\title{
OptLayer - Practical Constrained Optimization for Deep Reinforcement Learning in the Real World
}

\author{
Tu-Hoa Pham ${ }^{1}$, Giovanni De Magistris ${ }^{1}$ and Ryuki Tachibana ${ }^{1}$
}

\begin{abstract}
While deep reinforcement learning techniques have recently produced considerable achievements on many decision-making problems, their use in robotics has largely been limited to simulated worlds or restricted motions, since unconstrained trial-and-error interactions in the real world can have undesirable consequences for the robot or its environment. To overcome such limitations, we propose a novel reinforcement learning architecture, OptLayer, that takes as inputs possibly unsafe actions predicted by a neural network and outputs the closest actions that satisfy chosen constraints. While learning control policies often requires carefully crafted rewards and penalties while exploring the range of possible actions, OptLayer ensures that only safe actions are actually executed and unsafe predictions are penalized during training. We demonstrate the effectiveness of our approach on robot reaching tasks, both simulated and in the real world.
\end{abstract}

\section{INTRODUCTION}

Over the recent years, the rise of deep neural network architectures in multiple fields of science and engineering has come together with considerable achievements for deep reinforcement learning techniques, sometimes outperforming humans on high-dimensional problems such as Atari games [1] and Go [2]. The robotics field has also greatly benefitted from these advances, yielding impressive results on tasks otherwise difficult to model explicitly, e.g., manipulation from visual inputs [3] and robust locomotion on challenging terrains [4]. While visual understanding neural network pipelines can often be trained in an end-to-end fashion (e.g., inferring object poses directly from raw pixel information), robot motion is typically initialized in the real world from (partially) supervised learning, which supposes the availability of ground-truth data or expert demonstrations. In contrast, the discovery of robot motion behaviours from scratch is often limited to simulation due to time (to learn basic task features), safety (e.g., collisions), and other experimental constraints (e.g., resetting knocked-down obstacles to a chosen state). However, transferring control policies learned in simulation to reality remains a challenge, in particular due to model uncertainties (kinodynamics) [5] and variability between simulated and real observations [6]. Enabling deep reinforcement learning in the real world is thus crucial for tasks that cannot be learned in simulation and for which expert policies are not known.

In this paper, we propose a practical method to constrain neural network predictions to lie within a domain defined by safety constraints (see Fig. 1). Our work capitalizes on the state of the art in multiple areas of robotic control, optimization and reinforcement learning (Section III). We summarize

${ }^{1}$ IBM Research AI, Tokyo, Japan.

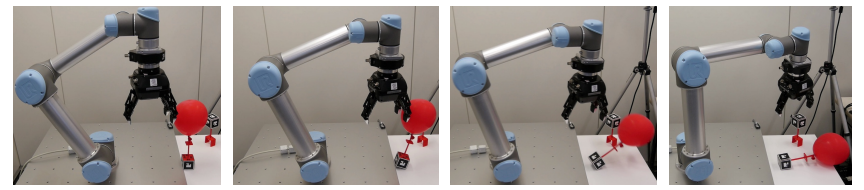

(a) Unconstrained action predictions can be dangerous to run on a real robot.

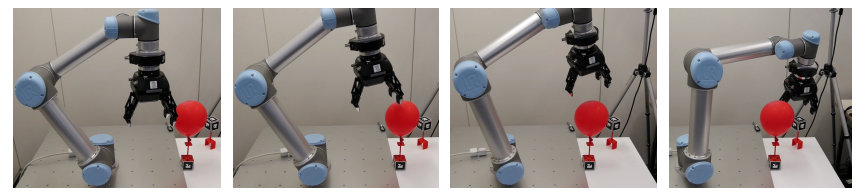

(b) Explicit constraints can be incorporated to guarantee safe robot actions.

Fig. 1. 3D reaching with obstacle avoidance using reinforcement learning.

the necessary technical background and important challenges for real-world reinforcement learning (Section III), in particular a strong dependency on the chosen reward structure.

- We augment a neural network architecture with a constrained optimization layer, OptLayer, that enforces arbitrary constraints on the predicted robot actions (Section IV). OptLayer is fully differentiable, enabling future end-to-end learning under safety constraints.

- We tackle the problem of safe reinforcement learning using OptLayer and propose a reward strategy that is simple to implement and readily compatible with existing policy optimization techniques (Section $\mathrm{V}$ ).

- Our approach makes exploration during training more efficient while always satisfying safety constraints. We demonstrate its effectiveness by learning 3D reaching with collision avoidance on an industrial manipulator, on tasks of increasing difficulty, both simulated and in the real world (Section VI.

Finally, we discuss challenges we encountered, current limitations and future extensions of our work (Section VII).

\section{RELATED WORK}

Robot motion is traditionally achieved by solving a multiparameter optimization problem yielding a sequence of states achieving a given task (e.g., track a given end effector trajectory) while respecting constraints motivated by safety and performance (e.g., joint limits). When constraints and task objectives are respectively linear and quadratic in the robot variables, these problems can be formulated and solved as quadratic programs (QP) [7]. Still, the resulting control policy can be unsatisfactory due to uncertainties on the robot kinodynamic model and sensor feedback (e.g., during locomotion, errors on center of mass coordinates can invalidate 
robot stability constraints), the chosen constraints being too conservative (making the motion suboptimal), or the task dynamics being non-trivial to model (e.g., finding the optimal trajectory to follow).

Reinforcement learning (RL) techniques have demonstrated impressive results in this context. In [1], convolutional neural networks (CNN) were trained to play Atari games using only pixels and game score as inputs, achieving humanlevel performance on multiple games without need for expert demonstrations or feature engineering. A guided policy search (GPS) method was proposed by [3] to predict motor torques for a PR2 robot from image and joint state inputs only, training the policy in a data-efficient manner using supervised learning (SL) and local controllers trained by RL. An overview and benchmark of modern RL algorithms for continuous control (e.g., for robotic applications) was presented in [8], along with valuable reference implementations released to the community. We thus chose to build our work upon the top-performing benchmarked algorithm, Trust-Region Policy Optimization (TRPO) [9], which we detail further in Section III-A. TRPO was notably used recently to discover dynamic locomotion behaviours such as crouching and jumping on simulated humanoids [4].

Applied in the real world, RL techniques require special attention to guarantee safe exploration during training, and safe execution at inference time. A thorough survey of safe RL was presented in [10], which classified techniques into two categories: those incorporating safety terms in the policy optimality criterion, and those modifying the exploration process using additional information. In particular, our work was inspired by [11], which proposed a new trust region method, Constrained Policy Optimization (CPO), to guarantee policy performance improvement with near-constraint satisfaction at each iteration, along with important results on convergence speed and worst-case constraint violation. Still, real-world robot experiments may require exact constraint satisfaction, though possibly at the cost of less (time and data) efficient training. Alternatively, constraints can be enforced on the neural network predictions directly: a priori, e.g., by placing limits on the range of possible actions before sampling [12]; or a posteriori, e.g., by ensuring that predictions do not send the robot's end effector outside of a chosen bounding sphere, reprojecting it inside if needed [13]. Our approach extends these ideas by reshaping the range of possible actions at each time step to ensure that resulting predictions always satisfy the exact safety constraints (not approximations thereof), during both training and inference. Our work also benefitted from [14], which proposed a neural network architecture to solve QP optimization problems in the context of SL, with applications to Sudoku puzzles. We extend this research towards RL for real-world systems with physical constraints.

\section{BACKGROUND AND MOTIVATION}

In this section, we summarize the RL algorithm our work builds upon, TRPO (Section III-A). We then consider the motivating example of a $2 \mathrm{D}$ reaching task (Section III-B) and illustrate the limitations of RL without explicit consideration of constraints (Section III-C), namely that learning a safe policy involves unsafe actions in the first place, with great sensitivity to the associated reward and cost structure.

\section{A. Reinforcement Learning Nomenclature}

We consider an infinite-horizon discounted Markov Decision Process (MDP) characterized by a tuple $\left(S, A, P, R, \rho_{0}, \gamma\right)$, with $S$ the set of states (e.g., robot joint configurations), $A$ the set of actions (e.g., motor commands), $P: S \times A \times S \rightarrow[0,1]$ the transition probability distribution to go from one state to another by taking a specific action, $R: S \times A \times S \rightarrow \mathbb{R}$ the function of associated rewards, $\rho_{0}: S \rightarrow[0,1]$ the initial state probability distribution, $\gamma \in[0,1)$ a discount factor. Denoting by $\pi: S \times A \rightarrow[0,1]$ a stochastic policy, the objective is typically to maximize the discounted expected return $\eta(\pi)=\underset{\tau}{\mathbb{E}}\left[\sum_{i=0}^{\infty} \gamma^{i} R\left(\mathbf{s}_{i}, \mathbf{a}_{i}, \mathbf{s}_{i+1}\right)\right]$, with $\tau=\left(\mathbf{s}_{0}, \mathbf{a}_{0}, \ldots\right)$ a state-action trajectory, $\mathbf{s}_{0} \sim \rho_{0}$, $\mathbf{a}_{i} \sim \pi\left(\cdot \mid \mathbf{s}_{i}\right)$ and $\mathbf{s}_{i+1} \sim P\left(\cdot \mid \mathbf{s}_{i}, \mathbf{a}_{i}\right)$. With the same notations, we denote by $Q_{\pi}$ the state-action value function, $V_{\pi}$ the value function and $A_{\pi}$ the advantage function:

$$
\begin{aligned}
Q_{\pi}\left(\mathbf{s}_{i}, \mathbf{a}_{i}\right) & =\underset{\mathbf{s}_{i+1}, \mathbf{a}_{i+1}, \ldots}{\mathbb{E}}\left[\sum_{l=0}^{\infty} \gamma^{l} R\left(\mathbf{s}_{i+l}, \mathbf{a}_{i+l}, \mathbf{s}_{i+l+1}\right)\right] \\
V_{\pi}\left(\mathbf{s}_{i}\right) & =\underset{\mathbf{a}_{i}, \mathbf{s}_{i+1}, \ldots}{\mathbb{E}}\left[\sum_{l=0}^{\infty} \gamma^{l} R\left(\mathbf{s}_{i+l}, \mathbf{a}_{i+l}, \mathbf{s}_{i+l+1}\right)\right], \\
A_{\pi}(\mathbf{s}, \mathbf{a}) & =Q_{\pi}(\mathbf{s}, \mathbf{a})-V_{\pi}(\mathbf{s})
\end{aligned}
$$

We consider in particular the case of a policy $\pi_{\theta}$ parameterized by a vector $\theta$ (e.g., neural network parameters). In TRPO, the policy $\pi_{\theta_{k}}$ is iteratively refined by solving the following optimization problem at each iteration $k$ :

$$
\begin{aligned}
& \theta_{k+1}=\underset{\theta}{\operatorname{argmax}} \underset{\mathbf{s} \sim \rho_{\theta_{k}}, \mathbf{a} \sim \pi_{\theta_{k}}}{\mathbb{E}}\left[\frac{\pi_{\theta}(\mathbf{a} \mid \mathbf{s})}{\pi_{\theta_{k}}(\mathbf{a} \mid \mathbf{s})} A_{\pi_{\theta_{k}}}(\mathbf{s}, \mathbf{a})\right] \\
& \text { such that } \underset{\mathbf{s} \sim \rho_{\theta_{k}}}{\mathbb{E}}\left[D_{\mathrm{KL}}\left(\pi_{\theta_{k}}(\cdot \mid \mathbf{s}) \| \pi_{\theta}(\cdot \mid \mathbf{s})\right)\right] \leq \delta_{\mathrm{KL}},
\end{aligned}
$$

with $\rho_{\theta}(\mathbf{s})=\sum_{i=0}^{\infty} \gamma^{i} P\left(\mathbf{s}_{i}=\mathbf{s}\right)$ the discounted state-visitation frequencies when actions are chosen according to $\pi_{\theta}, D_{\mathrm{KL}}$ the Kullback-Leibler divergence (measuring how much two probability distributions differ from each other), and $\delta_{\mathrm{KL}}$ a step size controlling how much the policy is allowed to change at each iteration. Eq. (4) is then solved numerically by sampling state-action trajectories following $\pi_{\theta_{k}}$ and averaging over samples. Our work builds on top of the OpenAI Baselines reference implementation for TRPQ ${ }^{1}$, in which a neural network $\mathcal{N}$ predicts state values jointly with actions. In the rest of the paper, we refer to it as a procedure UPDATENETWORK $\left(\mathcal{N},\left(\mathbf{s}_{i}, \mathbf{a}_{i}, r_{i}, v_{i}\right)_{i}\right)$ that iteratively optimizes $\mathcal{N}$ from sequences of state-action-reward-value tuples $\left(\mathbf{s}_{i}, \mathbf{a}_{i}, r_{i}, v_{i}\right)_{i=0, \ldots, N}$ over finite horizon $N$. 




(a) 2-DoF robot, target and obstacle.

\begin{tabular}{|c|c|}
\hline TYPE & REWARD \\
\hline$r_{\text {dist }}$ & $-d_{\mathrm{T}}$ \\
\hline$r_{\text {coll }}$ & $-20 \beta_{\text {coll }}$ \\
& $\begin{array}{c}\text { if collision } \\
\text { else } 0\end{array}$ \\
\hline$r_{\text {prox }}$ & $\begin{array}{c}2 \text { if } d_{\mathrm{T}} \leq 3 \mathrm{~cm} \\
\text { else } 0\end{array}$ \\
\hline
\end{tabular}

(b) 2D-reaching reward structure.
Fig. 2. 2D reacher description and rewards, with $d_{\mathrm{T}}$ the distance between target and end-effector and $\beta_{\text {coll }} \in\{1,5,10,50\}$ a penalization coefficient.

\section{B. Motivating Example}

We consider a 2-DoF robot with parallel revolute joints. The robot's base link is rigidly linked to the world such that it evolves in a plane $(\mathbf{x}, \mathbf{y})$ perpendicular to the gravity vector $-g \mathbf{z}$. Its two main links, upper arm and forearm, are of length $0.1 \mathrm{~m}$. The task consists in reaching a target point with the robot's end effector while avoiding a spherical obstacle, both on the plane $(\mathbf{x}, \mathbf{y})$. We denote by $\boldsymbol{\theta}=\left(\theta_{\text {shoulder }}, \theta_{\text {elbow }}\right)$ the robot's joint angles, with $\theta_{\text {shoulder }}$ not limited and $\theta_{\text {elbow }} \in$ $[-\pi,+\pi]$, and by $\dot{\theta}, \ddot{\theta}$ its joint velocities and accelerations, respectively. We depict the $2 \mathrm{D}$ reacher environment, $\mathcal{E}_{2 \mathrm{D}}$, in Fig. 2a. Each episode is initialized as follows:

1) The robot is reset to the initial state $\theta=\dot{\theta}=\ddot{\theta}=\mathbf{0}$.

2) The target's coordinates are randomly sampled following a uniform distribution of $\pm 0.27 \mathrm{~cm}$ along $\mathbf{x}$ and $\mathbf{y}$ (possibly out of reach for the robot).

3) The obstacle is initialized following the same distribution. If it collides at initialization with the robot, its position is re-sampled until it is no more the case.

We discretize time into $N=200$ steps of duration $\Delta T=$ $0.01 \mathrm{~s}$. The state vector $\mathbf{s}_{i}$ at step $i$, or time $t_{i}=i \Delta T$, is:

- $\mathbf{p}_{\mathrm{fa}}$ the 3D position of the forearm's base,

- $\theta_{\text {elbow }}$ the elbow joint angle,

- $\dot{\theta}$ the joint velocities,

- $\mathbf{p}_{\mathrm{ee}}$ the 3D position of the end effector,

- $\mathbf{p}_{\mathrm{t}}-\mathbf{p}_{\mathrm{ee}}$ the target position $\mathbf{p}_{\mathrm{t}}$ relative to the end effector,

- $\mathbf{p}_{\mathrm{o}}-\mathbf{p}_{\mathrm{ee}}$ the obstacle position $\mathbf{p}_{\mathrm{o}}$, also relative to $\mathbf{p}_{\mathrm{ee}}$.

The robot is controlled in position by providing, at step $i$, the desired joint position at step $i+1$. We define a zero-centered action $\mathbf{a}_{i}$ as the joint step $\Delta \boldsymbol{\theta}_{i}=\theta_{i+1}-\boldsymbol{\theta}_{i}$ to perform between two consecutive time steps. Finally, executing $\mathbf{a}_{i}$ in the environment yields three rewards, $r_{\text {dist }}$ (reward on low distance to target), $r_{\text {coll }}$ (collision penalization), $r_{\text {prox }}$ (bonus on proximity to target), detailed in Fig. $2 \mathrm{~b}$. We denote by $r_{i}$ the total reward at step $i$. In order to assess the sensitivity of RL to the reward structure, we parameterize the collision reward $r_{\text {coll }}$ with a coefficient $\beta_{\text {coll }} \in\{1,5,10,50\}$.

\section{Preliminary Results}

We implement the environment $\mathcal{E}_{2 \mathrm{D}}$ within the OpenAI Gym and Roboschool framework [15], which provides a unified interface to train and test neural network policies.

1https://github.com/openai/baselines
We use the physics engine within Roboschool to monitor external collisions, between any robot link and the obstacle, and auto-collisions, between the end effector and the base. Throughout this paper, we take as neural network $\mathcal{N}$ a simple multilayer perceptron (MLP) with two hidden layers of size 32 each. At each step $i$, the environment produces a state vector $s_{i}$, which is fed into $\mathcal{N}$ to produce an action and value pair $a_{i}, v_{i}$. Executing $a_{i}$ in $\mathcal{E}_{2 \mathrm{D}}$ results in a reward $r_{i}$ and an updated state $s_{i+1}$. We construct such sequences $\left(s_{i}, a_{i}, r_{i}, v_{i}\right)_{i=0, \ldots, N}$ over episodes of up to $N=200$ timesteps, interrupted in case of collision. On a 8-core computer, we train $\mathcal{N}$ on four instances of $\mathcal{E}_{2 \mathrm{D}}$ in parallel, with the TRPO procedure UPDATENETWORK of Section IIIA We report the evolution of the reward over 15000 episodes of training, for four values of $\beta_{\text {coll }}$, along with the cumulated number of collisions in Fig. 3. We observe the following:

- The reward evolution throughout training is greatly dependent on the collision penalization weight.

- With $\beta_{\text {coll }}=1$, the low penalty associated to collisions $r_{\text {coll }}=-20$ results in collision avoidance never being learnt. The optimal strategy consists in quickly going to the target to get the proximity reward $r_{\text {prox }}$ and possibly hitting the obstacle to start a new episode.

- Conversely, with $\beta_{\text {coll }}=50$, the large penalty $r_{\text {coll }}=$ -1000 results in fewer collisions but discourages exploration. The optimal strategy then consists in staying in the vicinity of the start pose and ignoring further targets.

- $\beta_{\text {coll }}=5$ and $10\left(r_{\text {coll }}=-100\right.$ and -200 , respectively $)$ are comparable in terms of rewards, but the latter results in half the number of collisions. This confirms the importance of carefully crafting the reward structure.

These results show that, without explicitly consideration of safety constraints during RL, it is difficult to learn a safe policy without violating such constraints many times. Overall, while we believe it is ultimately possible to design a reward structure that efficiently decreases collisions over time (e.g., empirically or by inverse reinforcement learning [16]), for the sake of RL in the real world, it is crucial to be able to learn to satisfy constraints without ever violating them.

\section{Constrained Optimization Layer}

\section{A. OptLayer Architecture}

With $\mathrm{x}$ a vector of $n_{\mathrm{x}}$ variables to optimize, we consider quadratic programs $(\mathrm{QP})$ of the form:

$$
\begin{aligned}
\min _{\mathbf{x}} & \frac{1}{2} \mathbf{x}^{T} \mathbf{P} \mathbf{x}+\mathbf{q}^{T} \mathbf{x} \\
\text { such that } & \mathbf{G x} \leq \mathbf{h} \\
\text { and } & \mathbf{A x}=\mathbf{b} .
\end{aligned}
$$

Eq. (6) is a quadratic objective in $\mathbf{x}$, with $\mathbf{P}$ a square matrix and $\mathbf{q}$ a vector of respective size $n_{\mathbf{x}} \times n_{\mathbf{x}}$ and $n_{\mathbf{x}}$; Eq (7) a set of $n_{\text {in }}$ linear inequalities, with $\mathbf{G}$ and $\mathbf{h}$ of respective size $n_{\text {in }} \times n_{\mathbf{x}}$ and $n_{\text {in }}$; and Eq (8) a set of $n_{\mathrm{eq}}$ linear equalities, with $\mathbf{A}$ and $\mathbf{b}$ of respective size $n_{\text {eq }} \times n_{\mathbf{x}}$ and $n_{\text {eq }}$. Our goal is to ensure that the action 

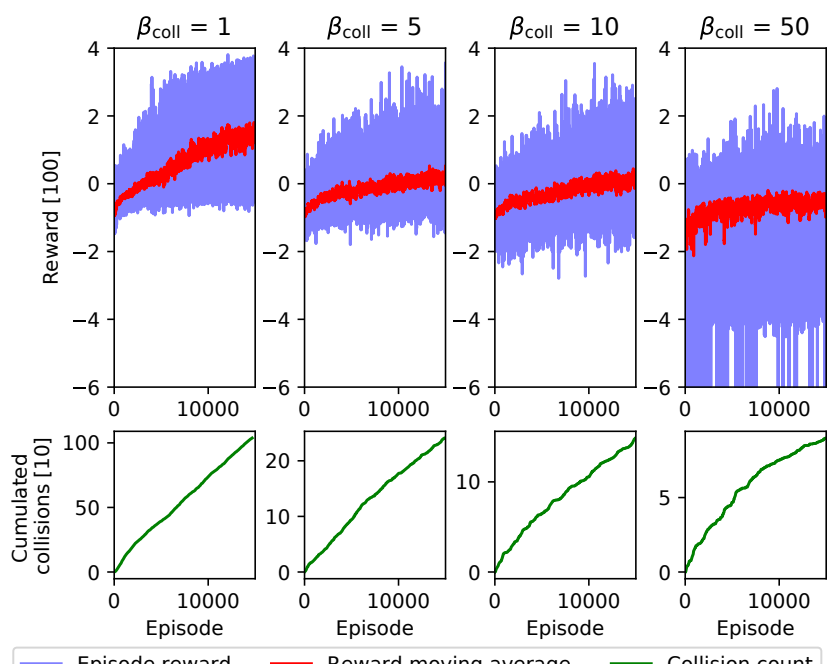

Fig. 3. Reward and collision count for unconstrained 2D reaching task with collision penalization coefficient $\beta_{\text {coll }} \in\{1,5,10,50\}$, averaged over three seeds. Moving average over 40 consecutive episode rewards.



(a) Prediction-correction architecture.

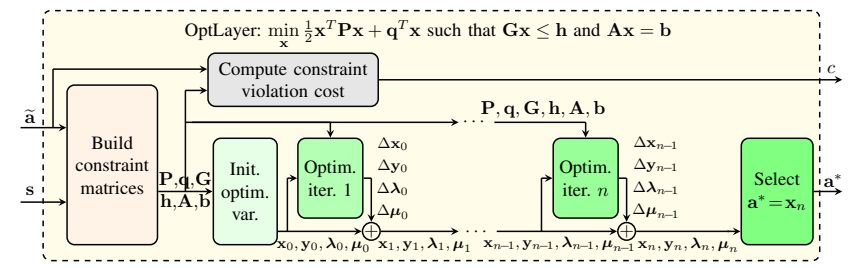

(b) OptLayer computes the safe action $\mathbf{a}^{*}$ that is closest to neural network prediction $\widetilde{\mathbf{a}}$, while quantifying how much the latter violates safety constraints.

Fig. 4. OptLayer constrained optimization pipeline.

$a_{t}$ predicted by a neural network $\mathcal{N}$ satisfies constraints such as Eqs. (7) and (8). In the following, we denote by $\widetilde{\mathbf{a}}_{i}$ the action predicted by the neural network $\mathcal{N}$ based on the current state $\mathbf{s}_{i}$. We propose a constrained optimization layer, OptLayer, that computes the action $\mathbf{a}_{i}^{*}$ that is closest to $\widetilde{\mathbf{a}}_{i}$ while also satisfying constraints depending on $\mathbf{s}_{i}$. We depict the overall architecture in Fig. 4a While multiple solutions are already available for solving QPs alone (as a forward pass), end-to-end neural network training requires a backward pass, in particular computing the gradients of the outputs with respect to the input parameters. We thus construct objective and constraint matrices as differentiable expressions of OptLayer's inputs $\mathbf{s}_{i}$ and $\widetilde{\mathbf{a}}_{i}$ (Section IVB]. We then solve the QP with an interior point method implemented as fully differentiable layers (Section IV-C).

\section{B. Quadratic Program Initialization}

Our first goal is to construct objective and constraint matrices as differentiable expressions of $\widetilde{\mathbf{a}}_{i}$ and $\mathbf{s}_{i}$. Special care is given to constraint matrices, which for robotic applications can be constant or affine in $\mathbf{s}_{i}$, require auxiliary state variables, or activate only under particular conditions. Although only inequality constraints appear in the following exposition, equality constraints are treated the same way.

1) Objective matrices: We minimize the (squared) $L^{2}$ distance between optimization variables $\mathbf{x}$ and prediction $\widetilde{\mathbf{a}}_{i}$ :

$$
\min _{\mathbf{x}} \frac{1}{2}\left\|\mathbf{x}-\widetilde{\mathbf{a}}_{i}\right\|^{2}=\frac{1}{2} \mathbf{x}^{T} \mathbf{x}-\widetilde{\mathbf{a}}_{i}^{T} \mathbf{x}+\frac{1}{2} \widetilde{\mathbf{a}}_{i}^{T} \widetilde{\mathbf{a}}_{i} .
$$

Since $\widetilde{\mathbf{a}}_{i}^{T} \widetilde{\mathbf{a}}_{i}$ does not depend on $\mathbf{x}$, it does not affect the optimum. Eq. (9) can thus be written as Eq. (6), with $\mathbf{P}=$ $\mathbf{I}_{n_{\mathbf{x}}}$ the identity matrix of size $n_{\mathbf{x}} \times n_{\mathbf{x}}$ and $\mathbf{q}=-\widetilde{\mathbf{a}}_{i}$.

2) Constant constraint matrices: In general, the constraints that affect a robot can be static or depend on its state. For the former, consider for example joint velocity limits:

$$
\dot{\theta}_{\min } \leq \dot{\theta} \leq \dot{\theta}_{\max },
$$

with $\dot{\theta}_{\min }, \dot{\theta}_{\max }$ velocity limits specified by the manufacturer (typically, $\dot{\theta}_{\min }=-\dot{\theta}_{\max }$ ). At step $i+1$, approximating joint velocities $\dot{\theta}_{i+1}$ with $\Delta \theta_{i} / \Delta T$ yields the following inequalities on action hypothesis $\mathrm{x}=\Delta \theta_{i}$, following Eq. (7):

$$
\left[\mathbf{I}_{n_{\mathbf{x}}}\right] \mathbf{x} \leq\left[\Delta T \dot{\boldsymbol{\theta}}_{\max }\right] \text { and }\left[-\mathbf{I}_{n_{\mathbf{x}}}\right] \mathbf{x} \leq\left[-\Delta T \dot{\boldsymbol{\theta}}_{\min }\right] .
$$

Eq (11) thus defines a set of constraint matrices $\mathbf{G}_{\mathrm{cst}}=$ $\left[\mathbf{I}_{n_{\mathbf{x}}},-\mathbf{I}_{n_{\mathbf{x}}}\right]^{T}$ and $\mathbf{h}_{\text {cst }}=\left[\Delta T \dot{\boldsymbol{\theta}}_{\max },-\Delta T \dot{\boldsymbol{\theta}}_{\min }\right]^{T}$ that are constant throughout the robot's motion.

3) Constraint matrices affine in the state vector: Consider now joint position limits $\theta_{\min }, \theta_{\max }$ :

$$
\theta_{\min } \leq \theta \leq \theta_{\max }
$$

In this case, the range of possible actions depends on the current joint positions. Since $\theta_{i+1}=\theta_{i}+\mathbf{x}$, we have:

$$
\left[\mathbf{I}_{n_{\mathbf{x}}}\right] \mathbf{x} \leq\left[\boldsymbol{\theta}_{\max }-\boldsymbol{\theta}_{i}\right] \text { and }\left[-\mathbf{I}_{n_{\mathbf{x}}}\right] \mathbf{x} \leq\left[\boldsymbol{\theta}_{i}-\boldsymbol{\theta}_{\min }\right] .
$$

Since $\theta_{i}$ is a member of the state vector $\mathbf{s}_{i}$, we can construct a selection matrix $\mathbf{H}_{\boldsymbol{\theta}}$ (with only 0 and \pm 1 ) such that $\left[-\theta_{i}, \boldsymbol{\theta}_{i}\right]^{T}=\mathbf{H}_{\boldsymbol{\theta}} \mathbf{s}_{i}$. Eq. (13) defines a set of constraint matrices that are constant or affine in $\mathbf{s}_{i}: \mathbf{G}_{\text {aff }}=\left[\mathbf{I}_{n_{\mathbf{x}}},-\mathbf{I}_{n_{\mathbf{x}}}\right]^{T}$ and $\mathbf{h}_{\text {aff }}=\mathbf{H}_{\boldsymbol{\theta}} \mathbf{s}_{i}+\left[\boldsymbol{\theta}_{\max },-\boldsymbol{\theta}_{\min }\right]^{T}$.

4) Auxiliary state parameters: In some cases, constraint matrices may not be formulated as a simple function of $\mathbf{s}_{i}$. Consider for example limits $\boldsymbol{\tau}_{\min }, \boldsymbol{\tau}_{\max }$ on joint torques $\boldsymbol{\tau}$ :

$$
\boldsymbol{\tau}_{\min } \leq \boldsymbol{\tau} \leq \boldsymbol{\tau}_{\max }
$$

With $\mathbf{q}, \dot{\mathbf{q}}, \ddot{\mathbf{q}}$ the robot's generalized coordinates (constant base pose and joint angles $\theta$ ), generalized velocities (zero base velocities $\mathbf{0}_{6}$ and joint velocities $\dot{\boldsymbol{\theta}}$ ) and generalized accelerations (zero base accelerations $\mathbf{0}_{6}$ and joint accelerations $\ddot{\theta})$, respectively, let $\mathbf{H}(\mathbf{q})$ be its mass matrix, $\mathbf{C}(\mathbf{q}, \dot{\mathbf{q}})$ the bias vector comprising Coriolis, centrifugal and gravitational forces, and $\mathbf{F}_{\text {base }}$ the 6-element vector of the external forces and torques exerted at the robot's (fixed) base. In the absence of other contact forces, the equations of motion are then:

$$
\mathbf{H}(\mathbf{q}) \ddot{\mathbf{q}}+\mathbf{C}(\mathbf{q}, \dot{\mathbf{q}})=\left[\begin{array}{c}
\mathbf{F}_{\text {base }} \\
\boldsymbol{\tau}
\end{array}\right] \text {. }
$$


The first 6 rows of Eq. (15) allow the computation of $\mathbf{F}_{\text {base }}$. We take $\mathbf{H}_{\boldsymbol{\tau}}(\mathbf{q})$ and $\mathbf{C}_{\boldsymbol{\tau}}(\mathbf{q}, \dot{\mathbf{q}})$ as the rows indexed 7 and onwards in $\mathbf{H}(\mathbf{q})$ and $\mathbf{C}(\mathbf{q}, \dot{\mathbf{q}})$, respectively, such that:

$$
\boldsymbol{\tau}=\mathbf{H}_{\boldsymbol{\tau}}(\mathbf{q}) \ddot{\mathbf{q}}+\mathbf{C}_{\boldsymbol{\tau}}(\mathbf{q}, \dot{\mathbf{q}}), \text { with } \ddot{\mathbf{q}}=\left[\begin{array}{c}
\mathbf{0}_{6} \\
\ddot{\theta}
\end{array}\right] \text {. }
$$

At step $i$, estimating $\ddot{\theta}_{i}$ by forward difference $\ddot{\theta}_{i}=\left(\dot{\theta}_{i+1}-\right.$ $\left.\dot{\theta}_{i}\right) / \Delta T$ and $\dot{\theta}_{i+1}=\left(\theta_{i+1}-\theta_{i}\right) / \Delta T=\mathrm{x} / \Delta T$ yields:

$$
\ddot{\theta}_{i}=\frac{\mathbf{x}}{\Delta T^{2}}-\frac{\dot{\theta}_{i}}{\Delta T} \text {. }
$$

Combining Eqs. (16) and (17) makes $\tau_{i}$ affine in $\mathrm{x}$ :

$$
\boldsymbol{\tau}_{i}=\frac{\mathbf{H}_{\boldsymbol{\tau}}\left(\mathbf{q}_{i}\right)}{\Delta T^{2}}\left[\begin{array}{c}
\mathbf{0}_{6} \\
\mathbf{x}
\end{array}\right]+\mathbf{C}_{\boldsymbol{\tau}}(\mathbf{q}, \dot{\mathbf{q}})-\frac{\mathbf{H}_{\boldsymbol{\tau}}\left(\mathbf{q}_{i}\right)}{\Delta T}\left[\begin{array}{c}
\mathbf{0}_{6} \\
\dot{\boldsymbol{\theta}}_{i}
\end{array}\right] .
$$

Since $\mathbf{H}_{\boldsymbol{\tau}}\left(\mathbf{q}_{i}\right)$ and $\mathbf{C}_{\boldsymbol{\tau}}\left(\mathbf{q}_{i}, \dot{\mathbf{q}}_{i}\right)$ can not be expressed as a trivial function (e.g., linear) of $\mathbf{s}_{i}$ in its current form, we adjust the environment $\mathcal{E}$ to compute them internally and append them to $\mathbf{s}_{i}$. Explicitly, we compute $\mathbf{H}_{\boldsymbol{\tau}}\left(\mathbf{q}_{i}\right)$ and $\mathbf{C}_{\boldsymbol{\tau}}\left(\mathbf{q}_{i}, \dot{\mathbf{q}}_{i}\right)$ from the robot's joint states and physical parameters using the KDL library [17] and store their elements as additional elements of $\mathbf{s}_{i}$. The joint torque constraints of Eq. (14) can then be expressed following Eq. (7), with constraint matrices $\mathbf{G}_{\text {aux }}$ and $\mathbf{h}_{\text {aux }}$ assembled from $\mathbf{s}_{i}$ (extended) directly.

5) Conditional constraint matrices: Finally, some constraints may be active only under some conditions. We consider the collision avoidance constraints of [18]. We denote by $\mathcal{L}_{l}$ a convex object associated to a robot link $l, \mathbf{p}_{l}$ the closest point between $\mathcal{L}_{l}$ and the environment, $d_{l}$ and $\mathbf{n}_{l}$ the corresponding distance and unit vector, oriented towards $\mathbf{p}_{l}$. If $d_{l}$ is smaller than a threshold $d_{M}$, named influence distance, then the following constraint is enabled:

$$
-\xi \frac{d_{l}-d_{m}}{d_{M}-d_{m}} \leq \dot{d}_{l}
$$

with $\dot{d}_{l}$ the distance velocity, $d_{m}$ a threshold security distance, and $\xi$ a positive velocity damping coefficient. Intuitively, Eq. (19) ensures that the distance $d_{l}$ between the robot and its environment cannot decrease faster than a chosen rate while $d_{m} \leq d_{l} \leq d_{M}$, and that $d_{l}$ strictly increases if $d_{l}<d_{m}$. Eq. (19) can be equivalently written as a constraint on $\dot{\mathbf{q}}$, with $\mathbf{J}\left(\mathbf{q}, \mathbf{p}_{l}\right)$ the Jacobian matrix of $\mathcal{L}_{l}$ at $\mathbf{p}_{l}$ when obstacles are static (otherwise it suffices to adjust $\dot{d}_{l}$ with their velocities):

$$
-\xi \frac{d_{l}-d_{m}}{d_{M}-d_{m}} \leq\left[\mathbf{n}_{l}^{T} \mathbf{J}\left(\mathbf{q}, \mathbf{p}_{l}\right)\right] \dot{\mathbf{q}} .
$$

Similarly to Section IV-B.4 the state vector $\mathbf{s}_{i}$ can be extended with the elements of $\mathbf{J}\left(\mathbf{q}, \mathbf{p}_{l}\right)^{T} \mathbf{n}_{l}$ to form constraint matrices $\mathbf{G}_{\text {cond }}$ and $\mathbf{h}_{\text {cond }}$. We also extend $\mathbf{s}_{i}$ with $d_{l}$ and denote by $\mathbf{H}_{d_{l}}$ the selection matrix such that $\mathbf{H}_{d_{l}} \mathbf{s}_{i}=d_{l}$. With $\mathbf{h}_{\text {test }}=\left[d_{M}\right]-\mathbf{H}_{d_{l}} \mathbf{s}_{i}$, conditional constraints and their activation can be expressed following:

$$
\text { If }\left\{\mathbf{0} \leq \mathbf{h}_{\text {test }}\right\} \text {, then enable }\left\{\mathbf{G}_{\text {cond }} \mathbf{x} \leq \mathbf{h}_{\text {cond }}\right\} \text {. }
$$

Note that activation condition $\left\{\mathbf{0} \leq \mathbf{h}_{\text {test }}\right\}$ can only depend on state $\mathbf{s}_{i}$, the action $\mathbf{x}$ being optimized subsequently.

\section{Quadratic Program Solving}

1) Assembling constraint matrices for batch solving: Using the notations of Section IV-B, we consider first a set of individual constraint matrices that are always active: $\mathbf{G}_{\text {cst }}, \mathbf{h}_{\text {cst }}, \mathbf{G}_{\text {aff }}, \mathbf{h}_{\text {aff }}, \mathbf{G}_{\text {aux }}, \mathbf{h}_{\text {aux }}$. We concatenate them into base constraint matrices $\mathbf{G}_{\text {base }}=\left[\mathbf{G}_{\mathrm{cst}}, \mathbf{G}_{\mathrm{aff}}, \mathbf{G}_{\mathrm{aux}}\right]^{T}$ and $\mathbf{h}_{\text {base }}=\left[\mathbf{h}_{\mathrm{cst}}, \mathbf{h}_{\mathrm{aff}}, \mathbf{h}_{\mathrm{aux}}\right]^{T}$ to be considered at every step. When solving only one QP at a time, conditional constraints matrices $\mathbf{G}_{\text {cond }}, \mathbf{h}_{\text {cond }}$ can be appended to the complete constraint matrices $\mathbf{G}, \mathbf{h}$ of Eq. (7) only when active, i.e.:

$$
\text { If } \begin{aligned}
\left\{\mathbf{0} \leq \mathbf{h}_{\text {test }}\right\}:\left\{\begin{array}{l}
\mathbf{G}=\left[\mathbf{G}_{\text {base }}, \mathbf{G}_{\text {cond }}\right]^{T}, \\
\mathbf{h}=\left[\mathbf{h}_{\text {base }}, \mathbf{h}_{\text {cond }}\right]^{T},
\end{array}\right. \\
\text { otherwise }:\left\{\begin{array}{l}
\mathbf{G}=\mathbf{G}_{\text {base }} \\
\mathbf{h}=\mathbf{h}_{\text {base }}
\end{array} .\right.
\end{aligned}
$$

However, deep neural networks are commonly trained using mini-batches of multiple input-output pairs in parallel. A technical consequence of parallel computing is that all elements processed together must be the same size. With $\mathbf{G}_{\text {sub }}$ (resp. $\mathbf{h}_{\text {sub }}$ ) a submatrix of $\mathbf{G}_{\text {base }}$ (resp. $\mathbf{h}_{\text {base }}$ ) of size that of $\mathbf{G}_{\text {cond }}$ (resp. $\mathbf{h}_{\text {cond }}$ ), we replace Eq. (23) with:

$$
\text { If not }\left\{\mathbf{0} \leq \mathbf{h}_{\text {test }}\right\}:\left\{\begin{array}{l}
\mathbf{G}=\left[\mathbf{G}_{\text {base }}, \mathbf{G}_{\text {sub }}\right]^{T} \\
\mathbf{h}=\left[\mathbf{h}_{\text {base }}, \mathbf{h}_{\text {sub }}\right]^{T}
\end{array} .\right.
$$

$\mathbf{G}$ and $\mathbf{h}$ being of constant size thus enables batch solving.

2) Iterative resolution: Provided objective and constraint matrices $\mathbf{P}, \mathbf{q}, \mathbf{G}, \mathbf{h}, \mathbf{A}, \mathbf{b}$, we implement the interior point method for solving QPs described in [7]. With $\mathrm{x}$ still denoting the variables to optimize, $\boldsymbol{\lambda}$ slack variables for the $\mathrm{QP}, \boldsymbol{\mu}$ and $\mathbf{y}$ dual variables respectively associated to the inequality and equality constraints, finding the optimum $\mathrm{x}^{*}$ amounts to solving a sequence of linear systems. First:

$$
\left[\begin{array}{ccc}
\mathbf{P} & \mathbf{G}^{T} & \mathbf{A}^{T} \\
\mathbf{G} & -\mathbf{I}_{n_{\text {in }}} & \mathbf{0}_{n_{\text {in }}, n_{\text {eq }}} \\
\mathbf{A} & \mathbf{0}_{n_{\text {eq }}, n_{\text {in }}} & \mathbf{0}_{n_{\text {eq }}, n_{\text {eq }}}
\end{array}\right]\left[\begin{array}{c}
\mathbf{x} \\
\boldsymbol{\mu} \\
\mathbf{y}
\end{array}\right]=\left[\begin{array}{c}
-\mathbf{q} \\
\mathbf{h} \\
\mathbf{b}
\end{array}\right] .
$$

We then use the solution to Eq. (25) to set $\mathbf{x}_{0}=\mathbf{x}, \mathbf{y}_{0}=\mathbf{y}$, and initialize $\boldsymbol{\lambda}_{0}$ and $\boldsymbol{\mu}_{0}$ depending on the value of $\boldsymbol{\mu}$. We thus obtain a starting point $\left(\mathbf{x}_{0}, \boldsymbol{\lambda}_{0}, \boldsymbol{\mu}_{0}, \mathbf{y}_{0}\right)$. The QP is then solved iteratively. For $\left(\mathbf{x}_{k}, \boldsymbol{\lambda}_{k}, \boldsymbol{\mu}_{k}, \mathbf{y}_{k}\right)$ at iteration $k$, with $\Lambda_{k}=\operatorname{diag}\left(\boldsymbol{\lambda}_{k}\right)$ and $\mathbf{M}_{k}=\operatorname{diag}\left(\boldsymbol{\mu}_{k}\right)$, update directions are computed by solving linear systems of the form [7]:

$$
\left[\begin{array}{cccc}
\mathbf{P} & \mathbf{0}_{n_{\text {in }}, n_{\text {in }}} & \mathbf{G}^{T} & \mathbf{A}^{T} \\
\mathbf{0}_{n_{\text {in }}, n_{\mathbf{x}}} & \mathbf{M}_{k} & \boldsymbol{\Lambda}_{k} & \mathbf{0}_{n_{\text {in }}, n_{\text {eq }}} \\
\mathbf{G} & \mathbf{I}_{n_{\text {in }}} & \mathbf{0}_{n_{\text {in }}, n_{\text {in }}} & \mathbf{0}_{n_{\text {in }}, n_{\text {eq }}} \\
\mathbf{A} & \mathbf{0}_{n_{\text {eq }}, n_{\text {in }}} & \mathbf{0}_{n_{\text {eq }}, n_{\text {in }}} & \mathbf{0}_{n_{\text {eq }}, n_{\text {eq }}}
\end{array}\right]\left[\begin{array}{c}
\Delta \mathbf{x}_{k} \\
\Delta \boldsymbol{\lambda}_{k} \\
\Delta \boldsymbol{\mu}_{k} \\
\Delta \mathbf{y}_{k}
\end{array}\right]=\mathbf{d}_{k},
$$

with $\mathbf{d}_{k}$ a vector depending on the current iteration $\left(\mathbf{x}_{k}, \boldsymbol{\lambda}_{k}, \boldsymbol{\mu}_{k}, \mathbf{y}_{k}\right)$ and QP matrices $\mathbf{P}, \mathbf{q}, \mathbf{G}, \mathbf{h}, \mathbf{A}, \mathbf{b}$. [14] showed that such problems could be efficiently solved on the GPU and released a public implementation using the PyTorch framework. In our custom implementation, we solved Eqs. 25) and 26 using linear system solvers provided within the Tensorflow framework. We iteratively update $\mathbf{x}_{k+1}=\mathbf{x}_{k}+\Delta \mathbf{x}_{k}, \boldsymbol{\lambda}_{k+1}=\boldsymbol{\lambda}_{k}+\Delta \boldsymbol{\lambda}_{k}, \boldsymbol{\mu}_{k+1}=\boldsymbol{\mu}_{k}+$ 


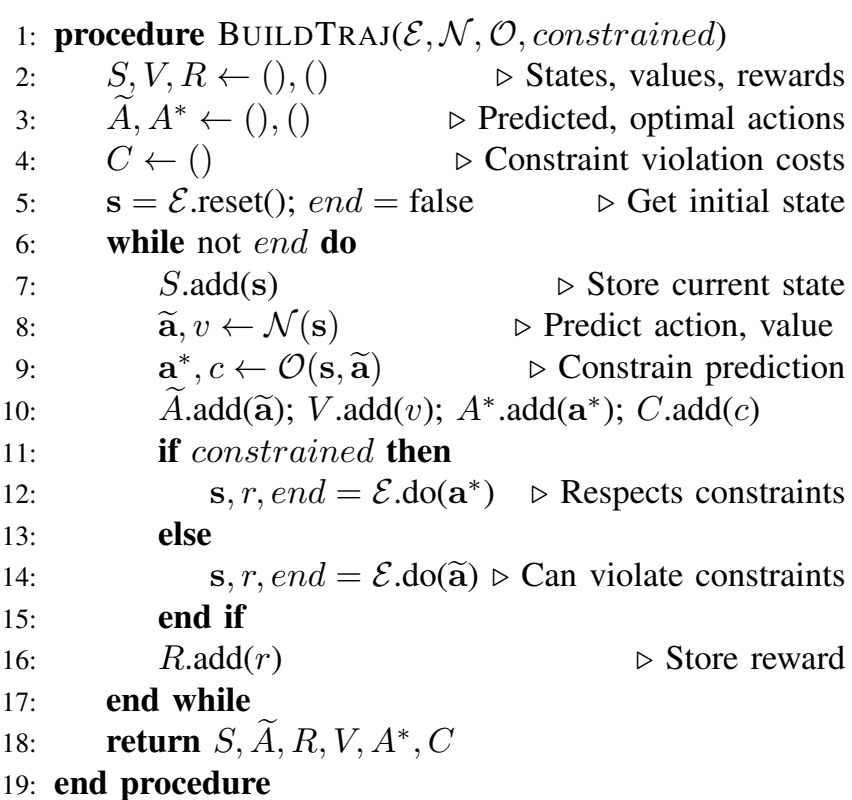

Fig. 5. Build a training trajectory for network $\mathcal{N}$ and optimization layer $\mathcal{O}$ in environment $\mathcal{E}$. If constrained $=$ false, execute raw predictions.

$\Delta \boldsymbol{\mu}_{k}, \mathbf{y}_{k+1}=\mathbf{y}_{k}+\Delta \mathbf{y}_{k}$, for $k=0, \ldots, k_{\max }$. While early stopping criteria can be employed for single QP solving, we take $k_{\max }$ constant for batch solving. Noting that the problems we solve typically converge in about 5 iterations, we empirically set $k_{\max }=10$. The final value for $\mathbf{x}$ is then outputted as the action $\mathbf{a}^{*}$ closest to the initial prediction $\widetilde{\mathbf{a}}$ that satisfies all safety constraints. We depict the complete OptLayer pipeline in Fig. 4b.

\section{CONSTRAined REINFORCEMENT LEARNing}

We propose a reward strategy that ensures that safety constraints are never violated throughout training and is readily compatible with existing RL methods, such as TRPO.

\section{A. Trajectory Sampling}

Recall from Section III-B that for unconstrained RL, we collect sequences of state-action-reward-value tuples $\left(\mathbf{s}_{i}, \widetilde{\mathbf{a}}_{i}, r_{i}, v_{i}\right)_{i=0, \ldots, N}$ by iteratively executing neural network predictions $\widetilde{\mathbf{a}}$ in the environment $\mathcal{E}$. Having established that executing unconstrained actions can be dangerous in the real world, we use OptLayer to produce corrected actions $\mathbf{a}^{*}$ and execute those instead in $\mathcal{E}$. Additionally, given constraint matrices $\mathbf{A}, \mathbf{b}, \mathbf{G}, \mathbf{h}$, it is possible to quantify how much unconstrained predictions $\widetilde{\mathbf{a}}$ violate those. We define equality and inequality violation $\cos t s c_{\mathrm{eq}}$ and $c_{\mathrm{in}}$, respectively:

$$
\begin{aligned}
c_{\mathrm{eq}} & =\|\mathbf{A} \widetilde{\mathbf{a}}-\mathbf{b}\|, \\
c_{\mathrm{in}} & =\left\|\max \left(\mathbf{G} \widetilde{\mathbf{a}}-\mathbf{h}, \mathbf{0}_{n_{\text {in }}}\right)\right\| .
\end{aligned}
$$

From Eq. (27), $c_{\text {eq }}$ increases any time $\mathbf{A} \widetilde{\mathbf{a}} \neq \mathbf{b}$, element-wise. Similarly, from Eq 28, , only elements such that $\mathbf{G} \widetilde{\mathbf{a}}>\mathbf{h}$ contribute to $c_{\text {in }}$ by taking the element-wise maximum with zero. In practice, the rows of $\mathbf{A}, \mathbf{b}$ (resp. $\mathbf{G}, \mathbf{h}$ ) can be scaled with an arbitrary non-zero (resp. positive) coefficient without changing the QP solution. We thus uniformize their individual contributions in $c_{\mathrm{eq}}$ (resp. $c_{\mathrm{in}}$ ) by normalization with the coefficients making each row of $\mathbf{A}$ (resp. $\mathbf{G}$ ) of unit norm. We denote by $c=c_{\mathrm{eq}}+c_{\mathrm{in}}$ the total constraint violation and compute it within OptLayer, together with $\mathbf{a}^{*}$. We define a procedure BUILDTRAJ in Fig. 5, that takes as inputs an environment $\mathcal{E}$, a neural network $\mathcal{N}$ with OptLayer $\mathcal{O}$, a flag constrained determining whether constrained or unconstrained actions are executed, and outputs states, raw predictions, rewards, values, corrected actions and constraint violation costs $\left(\mathbf{s}_{i}, \widetilde{\mathbf{a}}_{i}, r_{i}, v_{i}, \mathbf{a}_{i}, c_{i}\right)_{i}$. By setting constrained $=$ false, unconstrained actions are executed We only do so in simulation. For real-world RL, we set constrained $=$ true and only execute corrected actions.

\section{B. Policy Update Strategies}

With the procedure UPDATENETWORK described in Section III-A, we update the network $\mathcal{N}$ with sequences of the form $\left(\mathbf{s}_{i}, \mathbf{a}_{i}, r_{i}, v_{i}\right)_{i}$. We assess four ways to do so.

1) Unconstrained Predictions (UP): We run BUILDTRAJ with constrained $=$ false and update $\mathcal{N}$ with $\operatorname{UpdateNeTWORK}\left(\mathcal{N},\left(\mathbf{s}_{i}, \widetilde{\mathbf{a}}_{i}, r_{i}, v_{i}\right)_{i}\right)$, as in Section III-B. This is our baseline for unconstrained RL.

2) Constrained - learn Predictions (CP): We run BUILDTRAJ with constrained $=$ true and update $\mathcal{N}$ with UpdateNetwork $\left(\mathcal{N},\left(\mathbf{s}_{i}, \widetilde{\mathbf{a}}_{i}, r_{i}, v_{i}\right)_{i}\right)$. Note that different state sequences are produced compared to UP, as corrected (not predicted) actions are executed.

3) Constrained - learn Corrections (CC): We run BUILDTRAJ with constrained $=$ true and update $\mathcal{N}$ with Updatenetwork $\left(\mathcal{N},\left(\mathbf{s}_{i}, \mathbf{a}_{i}^{*}, r_{i}, v_{i}\right)_{i}\right)$.

4) Constrained - learn Predictions and Corrections (CPC): We run BUILDTRAJ with constrained = true and compute discounted rewards $\widetilde{r}_{i}$ using constraint violation costs $c_{i}: \tilde{r}_{i}=r_{i}-c_{i}$. We first update $\mathcal{N}$ with raw predictions and discounted rewards, UPDATENETWORK $\left(\mathcal{N},\left(\mathbf{s}_{i}, \widetilde{\mathbf{a}}_{i}, \widetilde{r}_{i}, v_{i}\right)_{i}\right)$. We update $\mathcal{N}$ a second time, using corrected actions and non-discounted rewards from initial sampling: $\operatorname{UPDATENETWORK}\left(\mathcal{N},\left(\mathbf{s}_{i}, \mathbf{a}_{i}^{*}, r_{i}, v_{i}\right)_{i}\right)$.

Intuitively, the $\mathrm{CP}$ policy update strategy amounts to considering OptLayer separately from the network and as part of the environment. In the CC strategy, we try to learn corrected actions directly, that can possibly greatly differ from initial neural network predictions. With CPC, we first associate raw predictions with discounted rewards before associating corrected actions with better rewards.

\section{EXPERIMENTS}

We now illustrate the application of OptLayer in the context of RL to learn 3D reaching tasks using a 6-DoF industrial manipulator (Section VI-A), both in simulation (Section VI-B) and in the real world (Section VI-C).

\section{A. 3D Reaching with Obstacle Avoidance}

We perform our experiments on a 6-DoF industrial manipulator (Universal Robots UR5), equipped with a forcetorque sensor (Robotiq FT-150) and a gripper (Robotiq 3Finger Adaptive Robot Gripper). Although we do not use 


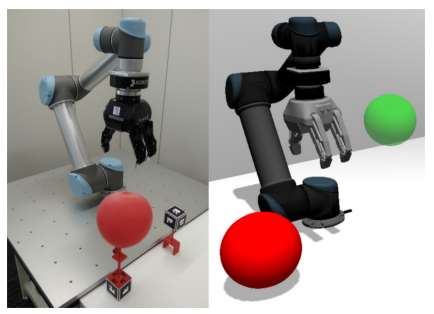

(a) UR5 robot, obstacle and target.

\begin{tabular}{|c|c|}
\hline TYPE & REWARD \\
\hline$r_{\text {dist }}$ & $-10 d_{\mathrm{T}}$ \\
\hline$r_{\text {coll }}$ & -100 \\
& if collision \\
& else 0 \\
\hline$r_{\text {prox }}$ & $\begin{array}{c}10 \text { if } d_{\mathrm{T}} \leq 5 \mathrm{~cm} \\
\text { else } 0\end{array}$ \\
\hline
\end{tabular}

(b) 3D-reaching reward structure.
Fig. 6. 3D reacher environment, with $d_{\mathrm{T}}$ the distance between target and gripper. In simulation, obstacle (red) and target (green) are floating spheres. In reality, we position them using handles with visual markers.

the latter to actually grasp objects in our experiments, we keep it for the sake of realism, making it even more crucial to preserve safety. We control the robot by directly sending desired trajectories through the Robot Operating System (ROS) middleware, in joint space, without making use of any safety mechanism available. We define a 3D reacher environment, $\mathcal{E}_{3 \mathrm{D}}$, where the goal is to send consecutive joint commands to reach a target point in 3D while avoiding collisions with the environment and the robot itself. At any step $i$, joint states $\boldsymbol{\theta}_{i}, \dot{\boldsymbol{\theta}}_{i}, \ddot{\boldsymbol{\theta}}_{i}$ and actions $\mathbf{a}_{i}=\boldsymbol{\theta}_{i+1}-\boldsymbol{\theta}_{i}$ are now of size 6 . The state vector $\mathbf{s}_{i}$ contains joint positions and velocities $\boldsymbol{\theta}_{i}, \dot{\theta}_{i}$, end effector position taken at the center of the finger ends $\mathbf{p}_{\mathrm{ee}, i}$, and target, obstacle $3 \mathrm{D}$ positions relative to $\mathbf{p}_{\mathrm{ee}, i}$. Each episode is run following the same procedure as the 2D case, except for one adjustment, motivated by real-robot training. At the end of each episode, we reset the robot by replaying the trajectory in reverse. This is important since going back from end to initial pose by joint space interpolation can lead to collisions in between. We set the maximum number of time steps to $N=100$, with duration $\Delta T=0.1 \mathrm{~s}$. We implement $\mathcal{E}_{3 \mathrm{D}}$ to enable parallel training on multiple physical robots or simulation instances (Gazebo).

\section{B. Evaluating Policy Update Strategies}

To evaluate the UP, CP, CC and CPC policy update strategies, we initialize safety constraint matrices as described in Section IV. While we could formulate them manually based on the specifications of our robot system (UR5 robot, forcetorque sensor, gripper), it is in fact possible to partially automate their definition. We leverage the availability of standardized robot description formats (e.g., URDF, MJCF) often directly released by robot manufacturers. We thus automatically generate constraint matrices for joint limits by simply parsing kinematic and physical parameters from such files. For collision constraints, we manually construct convex hulls for every robot link to enable fast distance computation [19]. We then automate the formulation of collision constraint matrices from a set of distances to monitor, of the form $\{$ (gripper, forearm), (elbow, obstacle $), \ldots\}$. In total, we enforce 36 joint constraints (position, velocity, torque) and 20 collision constraints (robot, floating obstacle, environment).

In simulation, we train neural networks on $\mathcal{E}_{3 \mathrm{D}}$ using UP, $\mathrm{CP}, \mathrm{CC}$ and $\mathrm{CPC}$. For each policy update strategy, we report rewards and collisions throughout 7000 episodes in Fig. 7a.

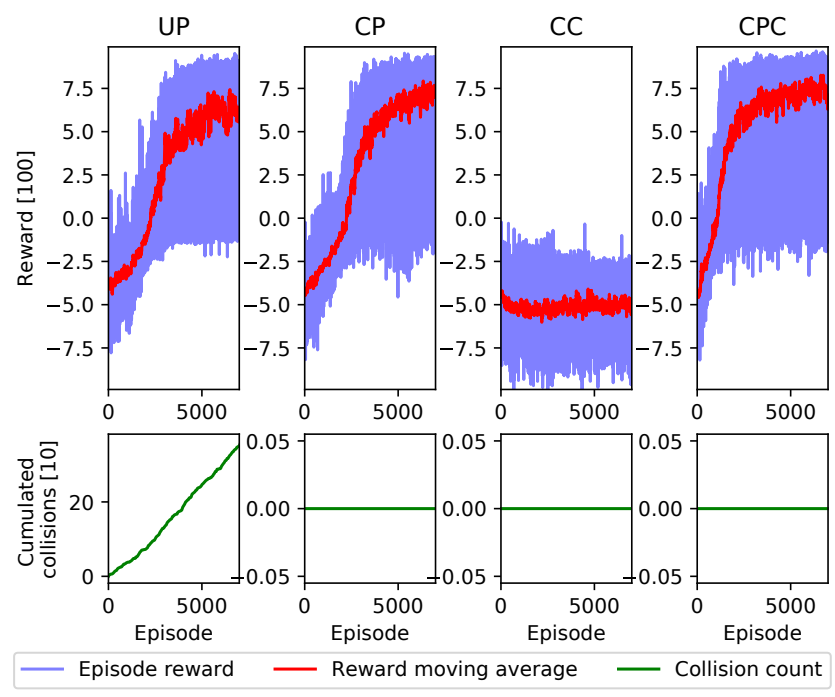

(a) Reward and collision count. Moving average over 40 consecutive episodes.

\begin{tabular}{|c|c|c|c|c|c|}
\cline { 2 - 6 } \multicolumn{1}{c|}{} & TOTAL Time & ST/EP & Time/ST & EP TO R & TiME TO R \\
\hline UP & $10 \mathrm{~h} 19 \mathrm{~min}$ & 88 & $60 \mathrm{~ms}$ & 5350 & $7 \mathrm{~h} 53 \mathrm{~min}$ \\
\hline $\mathrm{CP}$ & $12 \mathrm{~h} 28 \mathrm{~min}$ & 100 & $64 \mathrm{~ms}$ & 4950 & $8 \mathrm{~h} 49 \mathrm{~min}$ \\
\hline $\mathrm{CC}$ & $12 \mathrm{~h} 22 \mathrm{~min}$ & 100 & $64 \mathrm{~ms}$ & N/A & N/A \\
\hline $\mathrm{CPC}$ & $12 \mathrm{~h} 30 \mathrm{~min}$ & 100 & $64 \mathrm{~ms}$ & 3250 & $5 \mathrm{~h} 48 \mathrm{~min}$ \\
\hline
\end{tabular}

(b) Training time over 7000 episodes EP, steps $\mathrm{ST}$, average reward $\mathrm{R}=700$.

Fig. 7. Training results for $3 \mathrm{D}$ reaching with a 6-DoF robot, with policy update strategies UP (unconstrained) and CP, CC, CPC (constrained).

We observe the following. First, unconstrained training with $\mathrm{UC}$ results in the robot successfully learning to reach (top left), but not in effectively learning collision avoidance (bottom left). In contrast, using OptLayer successfully ensures that collisions never happen for all policy update strategies. In particular, $\mathrm{CP}$ realizes rewards that are comparable to UP while never putting the robot at risk. Recall that in $\mathrm{CP}$, from the perspective of the network, OptLayer can be seen as part of the environment, since the network is updated using raw predictions $\widetilde{\mathbf{a}}$ only. In constrast, updating the network using only corrected actions $\mathbf{a}^{*}$ within $\mathrm{CC}$ does not enable learning to reach. We believe this is due to $\mathbf{a}^{*}$ being initially too far from $\widetilde{\mathbf{a}}$ to be representative of the stochastic policy we optimize within TRPO, following Eq. (4). To do so successfully, CPC first updates the network with $\widetilde{\mathbf{a}}$ using discounted rewards prior to using $\mathbf{a}^{*}$. Subsequently, CPC appears to reach the performance of UP and CP with far fewer episodes. For example, a 700 average reward is reached in 5350 episodes by UP, 4950 by CP, 3250 by CPC. Detailed computation times (Fig. 7b) show that, though OptLayer induces some overhead ( $4 \mathrm{~ms}$ per step), the gain in terms of episodes makes CPC faster than UP even in terms of time.

\section{Real-World Experiments}

Having trained control policies in simulation, we execute them on a real robot. In these experiments, we position obstacle and target with respect to the robot using physical handles marked with 2D barcodes and tracked with an RGB camera. First, we consider a normal instance of $\mathcal{E}_{3 \mathrm{D}}$ with 

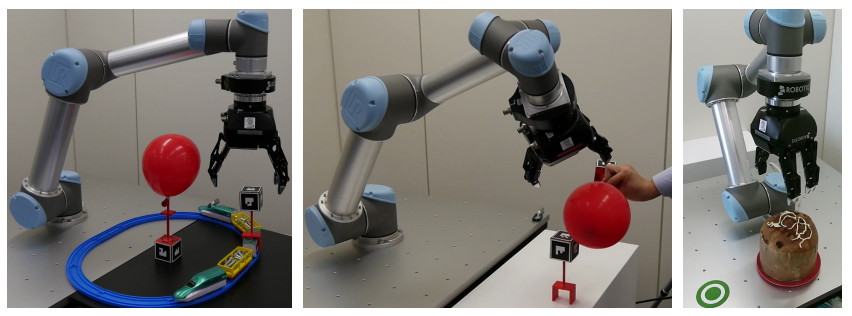

Fig. 8. From left to right: static obstacle, moving target; moving obstacle, moving target; static obstacle, static target learning on a real robot.

static target and obstacle. We execute the unconstrained policy learned with UP, $\mathcal{N}_{\mathrm{UP}}$, to reach a target behind a balloon (Fig. 1a). While reaching was successful, the balloon was knocked down, illustrating that collision avoidance was not effectively learned. We do not use $\mathcal{N}_{\text {UP }}$ further. In contrast, using the constrained policy learned with $\mathrm{CPC}, \mathcal{N}_{\mathrm{CPC}}$, results in successfully reaching the target while avoiding the balloon (Fig. 1b. As a qualitative example, we consider the case where the target is moving, carried by a toy train around a balloon (Fig. 8, left). Although $\mathcal{N}_{\text {CPC }}$ was not explicitly trained on this task, the target was successfully followed without hitting the balloon. We then consider the case where the obstacle is also moving. Through the collision avoidance constraints of Eq. (19), we verify that bringing the obstacle close to the robot forces the latter to move away from it. These results illustrate that combining control policies learned on specific tasks with OptLayer can help adapt to changing conditions, while always maintaining safety. Finally, although position control policies can be learned in simulation for $\mathcal{E}_{3 \mathrm{D}}$, real-world training can be necessary, e.g., for tasks involving force control [20] and physical interaction with the environment [13]. We thus learn a simplified version of $\mathcal{E}_{3 \mathrm{D}}$, in which the real robot has to reach a static target without touching a static obstacle. For ease of visualization, we use a cake for the latter (Fig. 8, right), never hitting it over $6 \mathrm{~h}$ of training. Our approach can thus be used to learn a robot control policy from scratch without active human supervision. We present our complete experimental results in the supplementary materia ${ }^{2}$

\section{Discussion AND Future Work}

Our work establishes that stochastic control policies can be efficiently coupled with constrained optimization to enable RL in the real world, where safety is crucial. Our method is simple to implement and readily compatible with existing RL techniques. We illustrated its application on neural network policies optimized with TRPO and demonstrated its effectiveness on a 3D reaching task with collision avoidance. Real-world robot experiments showed in particular that our system could accomodate reasonable changes in environment and task conditions, even without dedicated training.

Still, our work is subject to some limitations in its current implementation. While we showed that our approach could speed up training by better exploiting unsafe predictions,

2 https://www youtube. com/watch?v=71iBbk3VjwQ learning control policies from scratch may remain too timeconsuming even for simple tasks. We would thus like to investigate novel strategies for action space exploration tailored to robotics. Another limitation is that formalizing safety constraints can be difficult, e.g., under dynamic model uncertainties. Even when constraints are well defined, their activation may be contingent on the accuracy of other perception systems (e.g., vision). Our work would thus benefit from further advances in learning perception and control under uncertainties [5], [6]. Next, we intend to tackle other challenging robot learning and control tasks, such as bipedal locomotion and multi-agent collaboration. In the long term, we plan to extend our approach to problems besides robotics, such as decision-making systems for finance or healthcare.

\section{REFERENCES}

[1] V. Mnih, K. Kavukcuoglu, D. Silver, A. A. Rusu, J. Veness, M. G Bellemare, A. Graves, M. Riedmiller, A. K. Fidjeland, G. Ostrovski, et al., "Human-level control through deep reinforcement learning," Nature, 2015.

[2] D. Silver, A. Huang, C. J. Maddison, A. Guez, L. Sifre, G. Van Den Driessche, J. Schrittwieser, I. Antonoglou, V. Panneershelvam, M. Lanctot, et al., "Mastering the game of go with deep neural networks and tree search," Nature, 2016.

[3] S. Levine, C. Finn, T. Darrell, and P. Abbeel, "End-to-end training of deep visuomotor policies," J. Mach. Learn. Res., 2016.

[4] N. Heess, S. Sriram, J. Lemmon, J. Merel, G. Wayne, Y. Tassa, T. Erez, Z. Wang, A. Eslami, M. Riedmiller, et al., "Emergence of locomotion behaviours in rich environments," arXiv, 2017.

[5] P. Christiano, Z. Shah, I. Mordatch, J. Schneider, T. Blackwell, J. Tobin, P. Abbeel, and W. Zaremba, "Transfer from simulation to real world through learning deep inverse dynamics model," arXiv, 2016.

[6] J. Tobin, R. Fong, A. Ray, J. Schneider, W. Zaremba, and P. Abbeel, "Domain randomization for transferring deep neural networks from simulation to the real world," in Proc. IEEE-RSJ Int. Conf. Intell. Robot. Syst., 2017.

[7] J. Mattingley and S. Boyd, "Cvxgen: A code generator for embedded convex optimization," Optimization and Engineering, 2012.

[8] Y. Duan, X. Chen, R. Houthooft, J. Schulman, and P. Abbeel, "Benchmarking deep reinforcement learning for continuous control," in Proc. Int. Conf. Mach. Learn., 2016.

[9] J. Schulman, S. Levine, P. Abbeel, M. Jordan, and P. Moritz, "Trust region policy optimization," in Proc. Int. Conf. Mach. Learn., 2015.

[10] J. Garcia and F. Fernández, "A comprehensive survey on safe reinforcement learning," J. Mach. Learn. Res., 2015.

[11] J. Achiam, D. Held, A. Tamar, and P. Abbeel, "Constrained policy optimization," in Proc. Int. Conf. Mach. Learn., 2017.

[12] Y. F. Chen, M. Liu, M. Everett, and J. P. How, "Decentralized noncommunicating multiagent collision avoidance with deep reinforcement learning," in Proc. IEEE Int. Conf. Robot. Autom., 2017.

[13] S. Gu, E. Holly, T. Lillicrap, and S. Levine, "Deep reinforcement learning for robotic manipulation with asynchronous off-policy updates," in Proc. IEEE Int. Conf. Robot. Autom., 2017.

[14] B. Amos and J. Z. Kolter, "Optnet: Differentiable optimization as a layer in neural networks," in Proc. Int. Conf. Mach. Learn., 2017.

[15] G. Brockman, V. Cheung, L. Pettersson, J. Schneider, J. Schulman, J. Tang, and W. Zaremba, "Openai gym," arXiv, 2016.

[16] C. Finn, T. Yu, J. Fu, P. Abbeel, and S. Levine, "Generalizing skills with semi-supervised reinforcement learning," in Proc. Int. Conf. Learn. Repr., 2017.

[17] R. Smits, H. Bruyninckx, and E. Aertbeliën, "Kdl: Kinematics and dynamics library," http://www.orocos.org/kdl. 2011.

[18] F. Kanehiro, F. Lamiraux, O. Kanoun, E. Yoshida, and J.-P. Laumond, "A local collision avoidance method for non-strictly convex polyhedra," Robot. Sci. Syst., 2008.

[19] J. Pan, S. Chitta, and D. Manocha, "Fcl: A general purpose library for collision and proximity queries," in Proc. IEEE Int. Conf. Robot. Autom., 2012.

[20] T. Inoue, G. De Magistris, A. Munawar, T. Yokoya, and R. Tachibana, "Deep reinforcement learning for high precision assembly tasks," in Proc. IEEE-RSJ Int. Conf. Intell. Robot. Syst., 2017. 